TRANSACTIONS OF THE

AMERICAN MATHEMATICAL SOCIETY

Volume 353, Number 2, Pages 635-653

S 0002-9947(00)02674-X

Article electronically published on October 19, 2000

\title{
TWO-PARAMETER SPECTRAL AVERAGING AND LOCALIZATION FOR NON-MONOTONIC RANDOM SCHRÖDINGER OPERATORS
}

\author{
DIRK BUSCHMANN AND GÜNTER STOLZ
}

\begin{abstract}
We prove exponential localization at all energies for two types of one-dimensional random Schrödinger operators: the Poisson model and the random displacement model. As opposed to Anderson-type models, these operators are not monotonic in the random parameters. Therefore the classical one-parameter version of spectral averaging, as used in localization proofs for Anderson models, breaks down. We use the new method of two-parameter spectral averaging and apply it to the Poisson as well as the displacement case. In addition, we apply results from inverse spectral theory, which show that two-parameter spectral averaging works for sufficiently many energies (all but a discrete set) to conclude localization at all energies.
\end{abstract}

\section{INTRODUCTION}

While the first rigorous proofs of localization for one-dimensional random Schrödinger operators and their discrete counterparts were given earlier (e.g. [11] 22, 3]), it has been the results of Kotani on general ergodic potentials [18, 20] which have provided the deepest insights and become the basis of most subsequent work on onedimensional random operators. Kotani's results on Lyapunov exponents and their relation to spectral properties, combined with the method of spectral averaging or the use of Wegner estimates, have provided an elegant and widely applicable proof of one-dimensional localization; see for example [29, 21, 4, 32].

Spectral averaging was originally introduced in the context of rank one perturbations and thus applied to situations involving random boundary conditions 19 in dimension $d=1$ and the discrete Anderson model [29, 30], for both $d=1$ and $d>1$. Subsequently, it was understood that spectral averaging can be used on more general perturbations: 'rank one' can be replaced by 'monotonicity' - in form sense - and an unrestrictive compactness assumption. This lead to proofs of localization for continuum Anderson and related models, in both the one-dimensional and multidimensional cases; e.g. [21, 7, 8]. Other approaches to localization, in particular in higher dimension, rely on Wegner estimates and avoid spectral averaging; e.g. 9, 12, 15]. This also usually requires some sort of monotonicity in the random parameters.

Received by the editors October 2, 1998.

2000 Mathematics Subject Classification. Primary 81Q10, 34L40, 60H25, 47B80.

Key words and phrases. Random operators, localization, spectral averaging.

Research partially supported by NSF grant DMS-9706076.

(C)2000 American Mathematical Society 
There are, however, several physically important models where the monotonicity condition breaks down. Specifically, this is the case for the Poisson model, as well as for the random displacement model to be introduced below. The main results of this paper are extensions of the method of spectral averaging which allow us to prove localization at all energies for these models in dimension one. A partial result of this type, still based on a monotonicity property, was obtained for the Poisson model in 32 .

Previous results on localization under lack of monotonicity were given in two papers by Klopp [17, 16. In [17] a Wegner estimate for a multidimensional continuum Anderson model without sign-restriction on the single site potential is derived, while [16] provides a Wegner estimate for a semi-classical version of the multidimensional displacement model.

Here are the models together with the detailed assumptions which will be used hereafter in this text:

In both models we assume that the "single site potential" $f \in L_{\text {loc }}^{2}(\mathbb{R})$ is realvalued, $f \not \equiv 0$, with supp $f \subset[-s, s]$ for some $s>0$. Also, $(\Omega, \mathcal{F}, \mathbb{P})$ is a complete probability space with events $\mathcal{F}$ and probability measure $\mathbb{P}$.

The Displacement Model. Let $s<\frac{1}{2}$, and let $d_{n}: \Omega \rightarrow\left[-d_{\max }, d_{\max }\right], n \in \mathbb{Z}$, be independent and identically distributed random variables such that $d_{\max }+s<$ $\frac{1}{2}$. Assume also that the distribution measure of $d_{n}$ has a non-trivial absolutely continuous component. For $\omega \in \Omega$ let

$$
\begin{aligned}
H_{\omega}^{D} & =-\frac{d^{2}}{d x^{2}}+V_{\omega}^{D}(x) \\
& =-\frac{d^{2}}{d x^{2}}+\sum_{n \in \mathbb{Z}} f\left(x-n-d_{n}(\omega)\right),
\end{aligned}
$$

which is selfadjoint in $L^{2}(\mathbb{R})$ if defined as the closure of the corresponding operator on $C_{0}^{\infty}(\mathbb{R})$.

The $d_{n}$ describe random displacements of particles in a crystal, modeling the disorder introduced by temperature effects. The existence of an absolutely continuous component in their distribution (or even pure absolute continuity) makes sense from the viewpoint of physics.

The Poisson Model. This is the random Schrödinger operator

$$
\begin{aligned}
H_{\omega}^{P} & =-\frac{d^{2}}{d x^{2}}+V_{\omega}^{P} \\
& =-\frac{d^{2}}{d x^{2}}+\sum_{i} f\left(x-x_{i}(\omega)\right),
\end{aligned}
$$

where the $x_{i}(\omega)$ for $\omega \in \Omega$ are the points of a Poisson process on $\mathbb{R}$ with constant density $\alpha>0$. For almost every $\omega$ they can be labeled in a measurable way by $i \in \mathbb{Z} \backslash\{0\}$ such that

$$
\ldots<x_{-1}(\omega)<0<x_{1}(\omega)<x_{2}(\omega)<\ldots .
$$

They have the property that for every Borel set $B \subset \mathbb{R}$

$$
\mathbb{P}\left(\#\left\{i: x_{i}(\omega) \in B\right\}=n\right)=\frac{(\alpha|B|)^{n}}{n !} e^{-\alpha|B|},
$$


where $|\cdot|$ is Lebesgue measure. Also, if $B_{1} \cap B_{2}=\emptyset$, then the random variables $\#\left\{i: x_{i}(\omega) \in B_{1}\right\}$ and $\#\left\{i: x_{i}(\omega) \in B_{2}\right\}$ are independent. Setting for a moment also $x_{0}:=0$ (not a Poisson point), we define the random distances

$$
p_{n}=x_{n+1}-x_{n}, \quad n \in \mathbb{Z} .
$$

The $p_{n}$ are i.i.d. with distribution density $\alpha e^{-\alpha t}$ (for more details and references see [32]).

Since $f$ is locally $L^{2}$ and compactly supported we have $\mathbb{E}\left(V_{\omega}^{P}(0)^{2}\right)<\infty$ (e.g. 32]), and thus $H_{\omega}^{P}$ is almost surely essentially selfadjoint on $C_{0}^{\infty}(\mathbb{R})[14$.

The Poisson model is used to describe amorphous media, i.e. solids with extreme structural disorder.

$H_{\omega}^{X}$ is ergodic for both models $X=P$ and $X=D$, i.e. there is an ergodic dynamical system $\left\{T_{y}\right\}$ on $(\Omega, \mathcal{F}, \mathbb{P})$, where $y \in \mathbb{R}$ for $X=P$ and $y \in \mathbb{Z}$ for $X=D$, such that

$$
U_{y} V_{\omega}^{X} U_{-y}=V_{T_{y} \omega}^{X} \quad \text { for all } y \text { and } \omega .
$$

Here $\left(U_{y} f\right)(x)=f(x-y)$. This, in particular, implies that there are deterministic sets $\Sigma_{a c}^{X}, \Sigma_{s c}^{X}$ and $\Sigma_{p p}^{X}$ such that almost surely

$$
\sigma_{a c}\left(H_{\omega}^{X}\right)=\Sigma_{a c}^{X}, \quad \sigma_{s c}\left(H_{\omega}^{X}\right)=\Sigma_{s c}^{X}, \quad \sigma_{p p}\left(H_{\omega}^{X}\right)=\Sigma_{p p}^{X},
$$

where $\sigma_{a c}, \sigma_{s c}$ and $\sigma_{p p}$ denote the absolutely continuous, singular continuous and point spectrum (closure of the set of eigenvalues), respectively. For the general theory of ergodic operators, see the monographs [5] and [27].

The assumption $f \in L_{l o c}^{2}$ guarantees that the fundamental results of Kotani [18. 20] extend to the models considered here; see [14, Appendix B]. The generalizations of Kotani theory provided by Minami [23, 24] cover the case $f \in L_{l o c}^{1}, f \geq 0$. While all our methods presented below work for general $f \in L_{l o c}^{1}$, this does not seem to be covered by any of the known versions of Kotani theory (but probably it could be).

Since $f$ is compactly supported, it follows from the independence properties of the Poisson or displacement model that $H_{\omega}^{X}$ is non-deterministic in the sense of [23] (generalizing [18]). Thus Kotani theory implies that the Lyapunov exponent $\gamma^{X}(E)$ is positive for almost every $E \in \mathbb{R}$. This in turn has the consequence that $\Sigma_{a c}^{X}=\emptyset$.

Our main result here is that in addition $\Sigma_{s c}^{X}=\emptyset$, and therefore the spectrum for both the Poisson model and the displacement model is pure point with probability one:

Theorem 1.1. $\Sigma_{a c}^{P}=\Sigma_{s c}^{P}=\emptyset$, i.e. $H_{\omega}^{P}$ has pure point spectrum for almost every $\omega$. The eigenfunctions decay exponentially at the rate of the Lyapunov exponent.

Theorem 1.2. $\Sigma_{a c}^{D}=\Sigma_{s c}^{D}=\emptyset$, i.e. $H_{\omega}^{D}$ has pure point spectrum for almost every $\omega$. The eigenfunctions decay exponentially at the rate of the Lyapunov exponent.

To our knowledge, Theorem 1.2 provides the first proof of localization at all energies for the "pure" one-dimensional displacement model. In [16], Klopp studies the semi-classical model $-h^{2} \Delta+\sum_{n} f\left(x-n-d_{n}(\omega)\right)$ in arbitrary dimension. For a certain class of single site potentials $f$, in particular $f \leq 0, f \in C_{0}^{\infty}$ with sufficiently small support, and under suitable assumptions on the distribution of the $d_{n}$, in particular the existence of a density, localization is established in certain energy regions near the bottom of the spectrum if $h$ is sufficiently small. Some 
of the known proofs of localization for Anderson models extend to models with additional disorder in the displacement, but depend crucially on the existence of Anderson-type disorder; see for example [7, 17].

Theorem 1.1 extends the result of [32], where localization for the one-dimensional Poisson model was proven at positive energies.

The problems arising from non-monotonicity can be illustrated by the breakdown of the methods in 32 for energies $E<0$ : One of the basic approaches to localization is to use positivity of the Lyapunov exponent in combination with the method of spectral averaging. The result for the Poisson model in [32] is based on spectral averaging for the one-parameter family of operators

$$
H_{a}=-\frac{d^{2}}{d x^{2}}+V_{a}, \quad a \in(0, \infty),
$$

where $V_{a}(x)=V(x+a)$ for $x<-a, V_{a}(x)=0$ for $-a \leq x<a$, and $V_{a}(x)=V(x-a)$ for $a \leq x$. Here $V$ is a fixed potential. The family $H_{a}$ essentially arises by fixing all but one of the $p_{n}$ in $H_{\omega}^{P}$. For the Weyl-Titchmarsh spectral measures $\rho_{a}$ of $H_{a}$ it was shown in 32 that the "average measure"

$$
\int_{a_{1}}^{a_{2}} \rho_{a} d a
$$

is absolutely continuous in $(0, \infty)$ for arbitrary $0<a_{1}<a_{2}<\infty$. The proof of this fact uses monotonic $a$-dependence of eigenvalues of certain finite box operators. This is established in 32 by using Prüfer transform methods going back to Carmona's work, but can most easily be understood in terms of phase space volumes: Let $H_{a}^{N}$ be the restriction of $H_{a}$ to $L^{2}(-N-a, N+a)$ with, say, Dirichlet boundary conditions and fixed $N$; then the number of eigenvalues of $H_{a}^{N}$ below $E$ is approximately (e.g. 28])

$$
\int_{-N-a}^{N+a} \sqrt{\left(E-V_{a}\right)_{+}} d x
$$

which grows in $a$ for $E>0$, but remains constant for $E \leq 0$. In fact, the average measure (1.5) is not necessarily absolutely continuous in $(-\infty, 0)$ : To see this, fix an $E<0$ and choose $V$ on $(0, \infty)$ such that $-u^{\prime \prime}+V u=E u$ has a square-integrable solution $u_{+}$on $[0, \infty)$ with $u_{+}^{\prime}(0) / u_{+}(0)=\sqrt{|E|}$ (a suitable rectangular well will do). In a related way, we can choose $V$ on $(-\infty, 0)$ such that $-u^{\prime \prime}+V u=E u$ has a solution $u_{-}$on $(-\infty, 0]$ with $u_{-}^{\prime}(0) / u_{-}(0)=\sqrt{|E|}$. This choice of $V$ in the definition of $V_{a}$ provides an example in which $E$ is an eigenvalue of $H_{a}$ for every $a>0$, the corresponding eigenfunction $u_{a}$ being given by

$$
u_{a}(x)= \begin{cases}u_{-}(x+a), & x \leq-a, \\ c_{1} \exp (\sqrt{|E|} x), & -a<x<a, \\ c_{2} u_{+}(x-a), & x \geq a,\end{cases}
$$

with suitable constants $c_{1}$ and $c_{2}$. It follows for this example that $\rho_{a}(\{E\})>0$ for all $a$ and therefore $\int_{a_{1}}^{a_{2}} \rho_{a}(\{E\}) d a>0$. Thus the average measure has a non-trivial pure point component in $(-\infty, 0)$.

Similarly, fixing all but one $d_{n}$ in the displacement model leads to a family of the form $\tilde{H}_{a}=-d^{2} / d x^{2}+W(x)+f(x-a)$, where $W$ is supported in $\mathbb{R} \backslash\left[-\frac{1}{2}, \frac{1}{2}\right]$ and $a$ is sufficiently small so that $f(\cdot-a)$ is supported in $\left[-\frac{1}{2}, \frac{1}{2}\right]$. It is again possible to make non-trivial choices of $W$ and $f$ which give rise to a negative eigenvalue of $\tilde{H}_{a}$ which is stationary in $a$. 
Thus the traditional method of one-parameter spectral averaging does not work in either model. Here are our basic ideas to overcome the problem of nonmonotonicity:

(i) $H_{\omega}^{P}$ and $H_{\omega}^{D}$ depend on many parameters, which vary independently $\left(p_{n}\right.$ resp. $d_{n}$ ). If an eigenvalue $E$ is stationary under varying one parameter (as in the above examples), then it should be less likely (respectively happen for fewer $E$ 's) that it is stationary under independent variation of two or more parameters. This leads to the idea of using multiparameter spectral averaging (here: two parameters). This will allow us to exclude continuous spectrum from all but a discrete set of energies, which itself cannot carry a continuous spectrum.

(ii) "Monotonicity" of the model enters the proofs in [21] and 32] in the form of monotonic dependence of Prüfer angles on one of the random parameters (equivalent to monotonicity of eigenvalues of finite box operators). Lacking monotonicity, we will use the fact that it is sufficient to have analytic and non-constant dependence on the parameters. This shows local monotonicity away from a discrete set of parameters. To show non-constancy for the various models requires results from inverse spectral theory. Basically, these results say that if $f \neq 0$ in one of our models, then at least some solutions will satisfy different data than in the case $f=0$. We will collect these results in Section 3 .

(iii) It is sufficient to do spectral averaging locally in parameter intervals where the considerations from (ii) yield monotonicity. As long as these parameter values appear with positive probability (guaranteed by requiring absolutely continuous distributions), this leads to a proof of localization with positive probability. Nonrandomness of the spectral type implies almost sure localization.

In a separate publication we will use similar ideas to generalize known results for continuum one-dimensional Anderson type models of the form $H_{\omega}^{A}=-d^{2} / d x^{2}+$ $V_{\text {per }}(x)+\sum_{n} q_{n}(\omega) f(x-n)$, where $V_{\text {per }}$ is a periodic background potential and the $q_{n}$ are i.i.d. coupling constants. Almost sure localization at all energies can be shown for $H_{\omega}^{A}$ without sign restrictions on the single site potential $f$, i.e. without the monotonicity properties exploited in most of the existing work on Anderson models.

Our use of results from inverse spectral theory as given in Section 3 is closely related to ideas from scattering theory, as was pointed out to us by S. Molchanov and B. Simon. The use of scattering theoretic concepts in proofs of localization was discussed in [14] as well as in [26]. We plan to investigate these connections further in future work. In particular, this should allow us to extend our results to models with non-compactly supported single site potential $f$.

Some of the new ideas presented here, in particular the strategy of replacing monotonicity by analyticity and non-constancy, should also provide insights into related questions in higher dimensions. In particular, they may be useful in proving Wegner estimates for the Poisson model and for the 'non-semi-classical' version of the displacement model (i.e. without a small parameter $h$ as required in [16.

The proof of our main results is organized as follows: In Section 2 we use known facts from Kotani theory and the method of subordinate solutions to reduce the proofs of localization to results on two-parameter spectral averaging. Results from inverse theory, which will enter the proofs of spectral averaging, are provided in Section 3. Sections 4 and 5 make up the technical core of the paper, the proofs of spectral averaging for the Poisson model and displacement model, respectively. The details of this are somewhat easier for the Poisson model, while the method used 
for the displacement model in Section 5 has the advantage of supplying a second independent proof of localization for the Poisson case. We outline this briefly in Section 6 .

Acknowledgement. G. S. would like to thank Hans Volkmer from the University of Wisconsin at Milwaukee for a valuable discussion and for providing an elementary proof of Theorem 3.1 for the special case $q \in L^{2}$ in the early stages of this work.

\section{General Concepts}

Let $Q \in L_{l o c}^{1}$ be real-valued and such that $L=-d^{2} / d x^{2}+Q$ is the limit point at $\pm \infty$, and let $\rho$ be the corresponding Weyl-Titchmarsh spectral measure (cf. [6]), $\rho=\rho_{a c}+\rho_{s c}+\rho_{p p}$ the Lebesgue decomposition of $\rho$. Subordinacy theory [10] shows that

$$
\rho_{a c}\left(\mathbb{R} \backslash M_{a c}\right)=0, \quad \rho_{s c}\left(\mathbb{R} \backslash M_{s c}^{\prime}\right)=0,
$$

where

$$
\begin{aligned}
& M_{a c}=\{E \in \mathbb{R}: L u=E u \text { has no subordinate solution at }+\infty, \text { or } \\
&L u=E u \text { has no subordinate solution at }-\infty\}, \\
& M_{s c}^{\prime}=\left\{E \in \mathbb{R}: \begin{array}{l}
\text { There exists a solution of } L u=E u \text { which is } \\
\text { subordinate at } \left.+\infty \text { and at }-\infty, \text { but not in } L^{2}(\mathbb{R})\right\} .
\end{array}\right.
\end{aligned}
$$

Defining also

$$
\begin{aligned}
M_{s c}=\{E \in \mathbb{R}: & \text { There exists a solution } u_{+} \text {of } L u=E u \text { which is } \\
& \text { subordinate at }+\infty \text { and } u_{+} \notin L^{2}(0, \infty) \text {, or } \\
& \text { there exists a solution } u_{-} \text {of } L u=E u \text { which is } \\
& \text { subordinate at } \left.-\infty \text { and } u_{-} \notin L^{2}(-\infty, 0)\right\}
\end{aligned}
$$

one has $M_{s c}^{\prime} \subset M_{s c}$, and thus

$$
\rho_{s c}\left(\mathbb{R} \backslash M_{s c}\right)=0 \text {. }
$$

Theorem 2.1. Let $V_{\omega}$ be an ergodic potential such that the Lyapunov exponent $\gamma(E)$ is strictly positive for almost all $E \in \mathbb{R}$. Let $M_{a c}^{\omega}$ and $M_{s c}^{\omega}$ be the sets defined by (2.2) and (2.4) for $H_{\omega}=-d^{2} / d x^{2}+V_{\omega}$. Then

(a) $\left|M_{a c}^{\omega}\right|=0$ almost surely,

(b) $\left|M_{s c}^{\omega}\right|=0$ almost surely.

Remarks. (i) The idea of using subordinacy theory in proofs of localization is due to Minami 25].

(ii) Theorem 2.1(a) and (2.1) imply that $\sigma_{a c}\left(H_{\omega}\right)=\emptyset$ almost surely, i.e. $\Sigma_{a c}=\emptyset$. The proof of Theorem 2.1(a) below actually shows the Pastur-Ishii theorem $\Sigma_{a c} \subset$ $\{E: \gamma(E)=0\}^{e s s}$. That this follows easily from subordinacy theory was noted in [2].

Proof of Theorem 2.1. Positivity of the Lyapunov exponent implies by the theorem of Osceledec and Ruelle theorem (e.g. [5]) that for almost every $E$ there exist exponentially decaying solutions $u_{+}$and $u_{-}$of $H_{\omega} u=E u$ at $+\infty$ respectively $-\infty$ for a.e. $\omega$. By Fubini 'a.e. $E$ ' and 'a.e. $\omega$ ' can be exchanged. For these $\omega$ one has $\left|M_{a c}^{\omega}\right|=\left|M_{s c}^{\omega}\right|=0$, since an $E$ for which $u_{+}$and $u_{-}$as above exist is neither in $M_{a c}^{\omega}$ nor in $M_{s c}^{\omega}$ (this uses the fact that $L^{2}$-solutions are subordinate and that, up to a constant, at most one solution can be subordinate). 
For the rest of this section we look at the cases where $H_{\omega}$ is either $H_{\omega}^{D}$ or $H_{\omega}^{P}$. To this end, define the random variables $\xi_{n}, n \in \mathbb{Z}$, by

$$
\xi_{n}= \begin{cases}d_{n} & \text { in case of the displacement model, } \\ p_{n} & \text { in case of the Poisson model, }\end{cases}
$$

with distribution as given in Section 1.

For a given sequence $\xi=\left(\xi_{n}\right)$ let $H_{\xi}^{D}:=-d^{2} / d x^{2}+\sum_{n} f\left(x-n-\xi_{n}\right)$ and $H_{\xi}^{P}:=-d^{2} / d x^{2}+\sum_{i} f\left(x-x_{i}\right)$, where $x_{i}$ are the unique numbers determined by (1.4) with $\xi_{n}=p_{n}$ (and $x_{0}=0$ ). Let either $X=D$ or $X=P$, and let $M_{s c}^{\xi}$ be the set defined by (2.4) with $L=H_{\xi}^{X}$.

The existence of solutions as in (2.4) only depends on asymptotic properties of the potential. In particular, the set $M_{s c}^{\xi}$ does not depend on $\left(\xi_{0}, \xi_{1}\right)$, i.e. there exists a set $M_{s c}=M_{s c}\left(\left(\xi_{n}\right)_{n \in \mathbb{Z} \backslash\{0,1\}}\right)$ such that $M_{s c}^{\xi}=M_{s c}$ for all $\left(\xi_{0}, \xi_{1}\right)$.

The results of the remaining Sections 3 to 6 of this paper are summarized in

Theorem 2.2. Let $X=D$ or $X=P$. Then there exists a countable closed set $M \subset \mathbb{R}$ (only depending on the single site potential $f$ ) with the following property:

If $\left(\xi_{n}\right)_{n \in \mathbb{Z} \backslash\{0,1\}}$ is given such that $\left|M_{s c}\right|=0$ and $\mathbb{P}^{0,1}$ denotes the joint distribution of $\left(\xi_{0}, \xi_{1}\right)$, then for every $E_{0} \in \mathbb{R} \backslash M$ there exists $\varepsilon>0$ such that

$$
\mathbb{P}^{0,1}\left\{\sigma_{s c}\left(H_{\xi}^{X}\right) \cap\left(E_{0}-\varepsilon, E_{0}+\varepsilon\right)=\emptyset\right\}>0 .
$$

From here the proofs of our main results, Theorem 1.1 and Theorem 1.2, are completed as follows: By Theorem 2.1 one has $\left|M_{s c}(\omega)\right|=0$ for a.e. $\omega$, i.e. $\left|M_{s c}\right|=0$ for a.e. $\left(\xi_{n}\right)_{n \in \mathbb{Z} \backslash\{0,1\}}$. Theorem 2.2 , the independence of $\left(\xi_{0}, \xi_{1}\right)$ from $\left(\xi_{n}\right)_{n \in \mathbb{Z} \backslash\{0,1\}}$, and Fubini imply

$$
\mathbb{P}\left\{\sigma_{s c}\left(H_{\omega}^{X}\right) \cap\left(E_{0}-\varepsilon, E_{0}+\varepsilon\right)=\emptyset\right\}>0 .
$$

Since $\sigma_{s c}\left(H_{\omega}^{X}\right)=\Sigma_{s c}^{X}$ for a.e. $\omega$, this implies $\Sigma_{s c}^{X} \cap\left(E_{0}-\varepsilon, E_{0}+\varepsilon\right)=\emptyset$. This holds for arbitrary $E_{0}$ in the open set $\mathbb{R} \backslash M$; thus $\Sigma_{s c}^{X} \subset M$. The countable set $M$ cannot support any continuous spectrum; thus $\Sigma_{s c}^{X}=\emptyset$. Exponential decay of eigenfunctions at the Lyapunov rate holds almost surely by standard arguments, cf. [21, Theorem A1].

This leaves us with having to prove Theorem 2.2 which will be done separately for $X=P$ and $X=D$. In fact, we will find in both cases that Theorem 2.2 holds for a discrete set $M$.

\section{Some Inverse Theory}

The following Theorem 3.1 contains the key observation which will lead to the proof of Theorem 2.2 and thus of localization for $X=P$, i.e. the Poisson model (see Section 4). Theorem 3.1 combined with Corollary 3.2 will play an equally important role in the treatment of the displacement model in Section 5.

To prove Theorem 2.2 for $X=P$ we need to study the operators $H_{\xi}^{P}$ with all but the parameters $\xi_{0}=p_{0}$ and $\xi_{1}=p_{1}$ fixed. For this we introduce the two-parameter family $H_{a, b}=-d^{2} / d x^{2}+V_{a, b}$ of self-adjoint operators in $L^{2}(\mathbb{R})$, where for $a \geq 0$ and $\infty \geq b \geq 0$

$$
V_{a, b}(x)= \begin{cases}V(x), & x<0 \\ 0, & 0 \leq x<a \\ q(x-a), & a \leq x<a+D \\ 0, & a+D \leq x<a+D+b, \\ V(x-(a+D+b)), & a+D+b \leq x\end{cases}
$$


Self-adjointness (in the sense of SL-theory) is guaranteed by requiring $q \in L^{1}(0, D)$ and $V \in L_{l o c}^{1}(\mathbb{R})$ real-valued and such that the differential expression $-d^{2} / d x^{2}+V$ is limit point at $+\infty$ and $-\infty$. As in the counterexample given in Section 1, it is still possible to construct specific $V$ and $q$ such that for some $E<0$ there is a solution of $-u^{\prime \prime}+V_{a, b} u=E u$ which satisfies

(i) $u(x)=C e^{\sqrt{|E|} x}$ or $u(x)=C e^{-\sqrt{|E|} x}$ in $[0, a]$, and

(ii) $u(x)=C^{\prime} e^{\sqrt{|E|} x}$ or $u(x)=C^{\prime} e^{-\sqrt{|E|} x}$ in $[a+D, a+D+b]$, and

(iii) $u \in L^{2}(\mathbb{R})$.

Thus $E$ will be an eigenvalue of $H_{a, b}$ for all $a$ and $b$, excluding the possibility of proving spectral averaging. However, it turns out that for fixed $V$ and $q \neq 0$ this can happen for not more than a discrete set of negative E's. This is the content of Theorem 3.1 (note that (i) and (ii) above are equivalent to $u^{\prime}(a) / u(a) \in$ $\{-\sqrt{|E|}, \sqrt{|E|}\}$ and $\left.u^{\prime}(a+D) / u(a+D) \in\{-\sqrt{|E|}, \sqrt{|E|}\}\right)$.

Theorem 3.1. Let $q \in L^{1}(0, D)$ be real-valued with $q \neq 0$. Then the set $M_{0} \subset$ $(-\infty, 0]$ of all those $E \leq 0$ such that there exists a non-trivial solution $u$ of $-u^{\prime \prime}+$ $q u=E u$ on $[0, D]$ which satisfies

$$
\frac{u^{\prime}(0)}{u(0)} \in\{-\sqrt{|E|}, \sqrt{|E|}\} \quad \text { and } \quad \frac{u^{\prime}(D)}{u(D)} \in\{-\sqrt{|E|}, \sqrt{|E|}\}
$$

is discrete.

(3.2) represents four different "spectral parameter dependent boundary value problems" for $-u^{\prime \prime}+q u=E u$, according to the different sign combinations of $\pm \sqrt{|E|}$. The theorem says that if at least one of these boundary value problems has non-discrete negative "spectrum", then $q=0$. (Of course, for the sign combinations +- and -+ not even $q=0$ gives negative spectrum.) The case +- , and only this one, is obvious, since in this case $M_{0}$ coincides with the negative eigenvalues of $-d^{2} / d x^{2}+q$ on $L^{2}(\mathbb{R})$.

Proof. We get a periodic potential $Q$ on $\mathbb{R}$ by extending $q$ periodically. Let $T$ be the corresponding self-adjoint operator in $L^{2}(\mathbb{R})$. For $\alpha \in \mathbb{C}$ let $f_{\alpha}$ be the solution of $-u^{\prime \prime}+Q u=-\alpha^{2} u$ with initial conditions $f_{\alpha}(0)=1$ and $f_{\alpha}^{\prime}(0)=\alpha$. This gives entire functions $F^{-}(\alpha):=f_{\alpha}^{\prime}(D)-\alpha f_{\alpha}(D)$ and $F^{+}(\alpha):=f_{\alpha}^{\prime}(D)+\alpha f_{\alpha}(D)$. Suppose that $M$ is not discrete. It follows that $F^{-} \equiv 0$ or $F^{+} \equiv 0$. We are going to show that $Q=0$, i.e. $q=0$, which is a contradiction.

Case $F^{-}=0$ : We have $f_{\alpha}^{\prime}(D)=\alpha f_{\alpha}(D)$ for all $\alpha \in \mathbb{C}$. Thus all functions $f_{\alpha}$ are eigenfunctions of the corresponding transfer-matrix problem for $T$. For $\alpha \in \mathbb{R} \backslash\{0\}$, corresponding to negative energies, $f_{\alpha}$ is real-valued. Therefore the corresponding eigenvalue $f_{\alpha}(D)$ is real. This gives $\sigma(T) \subset[0, \infty)$ by Floquet theory.

For $\alpha \in i \mathbb{R} \backslash\{0\}$, corresponding to positive energies, $f_{\alpha}$ is not a multiple of a realvalued function. The corresponding eigenvalue (unless it is -1 or 1 ) therefore cannot be real. Again by Floquet theory, this yields $(0, \infty) \subset \sigma(T)$, i.e. $\sigma(T)=[0, \infty)$. By a classical result of Borg [1], this characterizes $Q=0$.

Case $F^{+} \equiv 0$ : This means that $f_{\alpha}^{\prime}(D)=-\alpha f_{\alpha}(D)$ for all $\alpha \in \mathbb{C}$, giving $f_{\alpha}^{\prime}(2 D)=$ $\alpha f_{\alpha}(2 D)$. By the arguments in the first case we again get $Q=0$.

Corollary 3.2. Let $q \in L^{1}(0, D)$ be real-valued, $q \neq 0, u_{0}(\cdot, \theta, E, q)$ the solution of the initial value problem

$$
-u^{\prime \prime}+q u=E u, \quad u(0)=\sin \theta, \quad u^{\prime}(0)=\cos \theta
$$


and $M_{1}$ the set of all those $E \in \mathbb{R}$ such that

$$
u_{0}^{\prime}(D, \theta, E, q)^{2}+E u_{0}(D, \theta, E, q)^{2}=\cos ^{2} \theta+E \sin ^{2} \theta
$$

for all $\theta \in[0, \pi)$. Then $M_{1}$ is a discrete set.

Note that (3.4) is equivalent to

$$
\int_{0}^{D} q(x) u_{0}(x, \theta, E, q) u_{0}^{\prime}(x, \theta, E, q) d x=0 .
$$

The corollary may be reformulated as follows: If the set of $E$ 's such that (3.4) or (3.5) holds for all $\theta$ has an accumulation point, then $q=0$.

Proof. Suppose that $M_{1}$ is not discrete. Then it follows from analyticity of (3.4) in $E$ (for fixed $\theta$ ) that $M_{1}=\mathbb{R}$. In particular, for arbitrary $E<0$ and $\theta=$ $\operatorname{arccot}( \pm \sqrt{|E|})$, i.e. $u^{\prime}(0) / u(0) \in\{ \pm \sqrt{|E|}\}$, it follows from (3.4) that $E u(D)^{2}+$ $u^{\prime}(D)^{2}=0$, i.e. $u^{\prime}(D) / u(D) \in\{ \pm \sqrt{|E|}\}$. By Theorem 3.1 this is only possible for $q=0$. Contradiction.

\section{Spectral Averaging for the Poisson Model}

Let $H_{a, b}=-d^{2} / d x^{2}+V_{a, b}$ be the two-parameter family of self-adjoint operators defined by (3.1). We assume that $q \neq 0$. By $\rho_{a, b}$ we denote the Weyl-Titchmarsh spectral measure for $H_{a, b}$. Theorem 2.2 in the case $X=P$ will follow from Theorem 4.1 below, which establishes spectral averaging for the family $H_{a, b}$ away from $M_{0} \cup\{0\}$, where $M_{0}$ is the set whose discreteness was proven in Theorem 3.1

Theorem 4.1. For fixed numbers $a_{2}>a_{1}>0$ and $b_{2}>b_{1}>0$ and arbitrary Borel sets $B \subset \mathbb{R}$ define

$$
\rho(B)=\int_{b_{1}}^{b_{2}} \int_{a_{1}}^{a_{2}} \rho_{a, b}(B) d a d b .
$$

Then the Borel measure $\rho$ is absolutely continuous on $\mathbb{R} \backslash\left(M_{0} \cup\{0\}\right)$.

At positive energies it will in fact not be necessary to average over both parameters simultaneously, i.e. it will turn out that $\int_{a_{1}}^{a_{2}} \rho_{a, b}(B) d a$ defines an absolutely continuous measure on $(0, \infty)$ for every $b$, which immediately implies that $\rho$ is absolutely continuous on $(0, \infty)$. This part of our result is basically identical to Proposition 3 of [32, where a slightly different one-parameter model was used. We will comment on this briefly at the end of the section and for now concentrate on the more involved proof of the fact that $\rho$ is absolutely continuous on $(-\infty, 0) \backslash M_{0}$.

For this it is sufficient to show the following: For every $E_{0} \in(-\infty, 0) \backslash M_{0}$ there exist $\varepsilon>0$ and $C>0$ such that

$$
\int f(E) d \rho(E) \leq C \int f(E) d E
$$

for every continuous non-negative $f$ with support in $\left(E_{0}-\varepsilon, E_{0}+\varepsilon\right)$. Thus we in fact establish local Lipshitz continuity of $\rho$ in $(-\infty, 0) \backslash M_{0}$.

The proof of (4.2) will use Prüfer variables. For the solution $u$ of $-u^{\prime \prime}+Q u=E u$ with $u(c)=\sin \theta$ and $u^{\prime}(c)=\cos \theta$ let

$$
r_{c}(x, \theta, E, Q)=\left(u(x)^{2}+u^{\prime}(x)^{2}\right)^{1 / 2}
$$


be the Prüfer amplitude and

$$
\phi_{c}(x, \theta, E, Q)=\arctan \frac{u(x)}{u^{\prime}(x)} \quad\left(\operatorname{or} \operatorname{arccot} \frac{u^{\prime}(x)}{u(x)}\right)
$$

be the Prüfer angle. Uniqueness of $\phi_{c}$ is achieved by requiring that $\phi_{c}(c, \theta, E, Q)=\theta$ and that $\phi_{c}(\cdot, \theta, E, Q)$ is continuous.

The estimate (4.2) will be a consequence of

Lemma 4.2. For each $E_{0} \in(-\infty, 0) \backslash M_{0}$ there are $\varepsilon>0$ and $C=C\left(a_{1}, a_{2}, b_{1}, b_{2}\right)$ $>0$ such that

$$
\int_{b_{1}}^{b_{2}} \int_{a_{1}}^{a_{2}} r_{0}\left(a+D+b+N, \theta, E, V_{a, b}\right)^{-2} d a d b \leq C
$$

uniformly in $N>0, \theta \in[0, \pi)$ and $E \in\left[E_{0}-\varepsilon, E_{0}+\varepsilon\right]$.

Proof. The definition of the Prüfer variables yields

$$
\begin{aligned}
& r_{0}\left(a+D+b+N, \theta, E, V_{a, b}\right)^{-2} \\
& \quad=r_{0}(a, \theta, E, 0)^{-2} r_{a}\left(a+D+b+N, \phi_{0}(a, \theta, E, 0), E, V_{a, b}\right)^{-2} \\
& \quad \leq C_{1} r_{0}\left(D+b+N, \phi_{0}(a, \theta, E, 0), E, V_{0, b}\right)^{-2}
\end{aligned}
$$

locally uniformly in $(a, \theta, E)$. Here we used the fact that $r_{0}$ does not vanish and is continuous in $(a, \theta, E)$.

In order to get an estimate for the $a$-integral in 4.3) we would like to use (4.4) and substitute $\alpha(a):=\phi_{0}(a, \theta, E, 0)$ for $a$. The problem arises that $\alpha(a)$ is stationary in $a$ for $\theta= \pm \operatorname{arccot} \sqrt{|E|}$, causing a singularity of the Jacobian. This leads to the necessity of a different estimate for $r_{0}\left(a+D+b+N, \theta, E, V_{a, b}\right)^{-2}$ if $\theta$ is close to $\pm \operatorname{arccot} \sqrt{|E|}$ (see (4.11) below). For these $\theta$ we know by Theorem 3.1 (and a continuity argument) that $\phi_{0}\left(a+D, \theta, E, V_{a, b}\right) \neq \pm \operatorname{arccot} \sqrt{|E|}$, since we only study energies $E \in(-\infty, 0) \backslash M_{0}$. Thus, in this case, $\phi_{0}\left(a+D+b, \theta, E, V_{a, b}\right)$ is non-stationary in $b$, which will allow us to estimate (4.3) by exchanging the order of integration, i.e. integrating over $b$ first. Here are the details:

From $E_{0} \in(-\infty, 0) \backslash M_{0}$ it follows that

$$
\phi_{0}\left(D, \pm \operatorname{arccot} \sqrt{\left|E_{0}\right|}, E_{0}, q\right) \notin\left\{ \pm \operatorname{arccot} \sqrt{\left|E_{0}\right|}\right\}+\pi \mathbb{Z} .
$$

This and the continuity of $\phi_{0}(D, \theta, E, q)$ in $\theta$ and $E$ imply the existence of $\varepsilon>0$ such that

$$
\phi_{0}(D, \theta, E, q) \notin\{ \pm \operatorname{arccot} \sqrt{|E|}\}
$$

for $E \in\left[E_{0}-\varepsilon, E_{0}+\varepsilon\right]$ and $\operatorname{dist}(\theta,\{ \pm \operatorname{arccot} \sqrt{|E|}\}) \leq \varepsilon$. From the "phase space" structure of the solutions of $-u^{\prime \prime}=E u$ for $E<0$ (in particular the asymptotic character of the lines $u^{\prime}=\sqrt{|E|} u$ and $u^{\prime}=-\sqrt{|E|} u$ ) it follows that there exists an $\varepsilon^{\prime}=\varepsilon^{\prime}\left(a_{2}\right)>0$ such that

$$
\left|\phi_{0}(a, \theta, E, 0)+\operatorname{arccot} \sqrt{|E|}\right| \leq \varepsilon
$$

if $E \in\left[E_{0}-\varepsilon, E_{0}+\varepsilon\right], a \in\left[a_{1}, a_{2}\right]$ and $|\theta+\operatorname{arccot} \sqrt{|E|}| \leq \varepsilon^{\prime}$, and such that

$$
\left|\phi_{0}(a, \theta, E, 0)-\operatorname{arccot} \sqrt{|E|}\right| \leq \varepsilon
$$


if $E \in\left[E_{0}-\varepsilon, E_{0}+\varepsilon\right], a \in\left[a_{1}, a_{2}\right]$ and $|\theta-\operatorname{arccot} \sqrt{|E|}| \leq \varepsilon^{\prime}$.

For fixed but arbitrary $E \in\left[E_{0}-\varepsilon, E_{0}+\varepsilon\right]$ the estimate (4.3) will now be shown separately for $\theta \in T_{1}=T_{1}(E)$ and $\theta \in T_{2}=T_{2}(E)$, where

$$
\begin{aligned}
& T_{1}:=\left\{\theta:|\theta-\operatorname{arccot} \sqrt{|E|}| \geq \varepsilon^{\prime} \text { and }|\theta+\operatorname{arccot} \sqrt{|E|}| \geq \varepsilon^{\prime}\right\}, \\
& T_{2}:=\left\{\theta:|\theta-\operatorname{arccot} \sqrt{|E|}| \leq \varepsilon^{\prime} \text { or }|\theta+\operatorname{arccot} \sqrt{|E|}| \leq \varepsilon^{\prime}\right\} .
\end{aligned}
$$

First assume that $\theta \in T_{1}$.

Then $\frac{d}{d a} \phi_{0}(a, \theta, E, 0)=\cos ^{2} \phi_{0}+E \sin ^{2} \phi_{0} \neq 0$ for all $a$. Thus by continuity

$$
\left|\frac{d}{d a} \phi_{0}(a, \theta, E, 0)\right| \geq C_{2}>0
$$

uniformly in $E \in\left[E_{0}-\varepsilon, E_{0}+\varepsilon\right], \theta \in T_{1}$ and $a \in\left[a_{1}, a_{2}\right]$.

This can be used to substitute $\alpha(a):=\phi_{0}(a, \theta, E, 0)$ for $a$ after integrating (4.4). Since $\alpha\left(\left[a_{1}, a_{2}\right]\right) \subset\left[\alpha_{1}, \alpha_{2}\right]$ locally uniformly in $(\theta, E)$, it follows that

$$
\begin{aligned}
\int_{a_{1}}^{a_{2}} & r_{0}\left(a+D+b+N, \theta, E, V_{a, b}\right)^{-2} d a \\
& \leq \frac{C_{1}}{C_{2}} \int_{\alpha_{1}}^{\alpha_{2}} r_{0}\left(D+b+N, \alpha, E, V_{0, b}\right)^{-2} d \alpha \\
& \leq \frac{C_{1}}{C_{2}}\left(\left|\alpha_{2}-\alpha_{1}\right|+\pi\right) .
\end{aligned}
$$

Here the last step follows by subdividing $\left[\alpha_{1}, \alpha_{2}\right]$ into intervals of length $\leq \pi$ and using the well known formula

$$
\int_{\theta}^{\theta+\pi} r_{0}(x, \alpha, E, Q)^{-2} d \alpha=\pi \text { for all } \theta, x, E, Q
$$

see e.g. Corollary 12 of [32]. Thus

$$
\int_{b_{1}}^{b_{2}} \int_{a_{1}}^{a_{2}} r_{0}(\ldots)^{-2} d a d b \leq \frac{C_{1}}{C_{2}}\left(b_{2}-b_{1}\right)\left(\left|\alpha_{2}-\alpha_{1}\right|+\pi\right),
$$

which completes the proof of Lemma 4.2 for the case $\theta \in T_{1}$.

In the remaining case $\theta \in T_{2}$ we will do the $b$-integration in (4.3) first. We start by estimating

$$
\begin{aligned}
r_{0}(a+ & \left.D+b+N, \theta, E, V_{a, b}\right)^{-2} \\
= & r_{0}\left(a+D+b, \theta, E, V_{a, b}\right)^{-2} \\
& \times r_{a+D+b}\left(a+D+b+N, \phi_{0}\left(a+D+b, \theta, E, V_{a, b}\right), E, V_{a, b}\right)^{-2} \\
\leq & C_{3} r_{0}\left(N, \phi_{0}\left(a+D+b, \theta, E, V_{a, \infty}\right), E, V\right)^{-2}
\end{aligned}
$$

locally uniformly in $(a, b, \theta, E)$. In order to substitute $\beta=\phi_{0}\left(a+D+b, \theta, E, V_{a, \infty}\right)$ for $b$ we will use the fact that

$$
\frac{d \beta}{d b}(b)=\cos ^{2} \phi_{0}\left(a+D+b, \theta, E, V_{a, \infty}\right)+E \sin ^{2} \phi_{0}\left(a+D+b, \theta, E, V_{a, \infty}\right)
$$

and that by "additivity" of the Prüfer angle

$$
\phi_{0}\left(a+D+b, \theta, E, V_{a, \infty}\right)=\phi_{0}\left(b, \phi_{0}\left(D, \phi_{0}(a, \theta, E, 0), E, q\right), E, 0\right) .
$$


By (4.5) and (4.6) or (4.7), $\phi_{0}\left(D, \phi_{0}(a, \theta, E, 0), E, q\right) \notin\{ \pm \operatorname{arccot} \sqrt{|E|}\}$ for $E \in$ $\left[E_{0}-\varepsilon, E_{0}+\varepsilon\right], \theta \in T_{2}$ and $a \in\left[a_{1}, a_{2}\right]$. Thus by (4.13) we get

$$
\phi_{0}\left(a+D+b, \theta, E, V_{a, \infty}\right) \notin\{ \pm \operatorname{arccot} \sqrt{|E|}\} \quad \text { for } b \in\left[b_{1}, b_{2}\right] .
$$

(4.12) yields $\frac{d \beta}{d b} \neq 0$, and thus by continuity

$$
\left|\frac{d \beta}{d b}\right| \geq C_{4}>0
$$

uniformly in the parameter range considered for $(E, \theta, a, b)$. From here the proof of Lemma 4.2 for $\theta \in T_{2}$ is completed as in the case $\theta \in T_{1}$, integrating over $b$ first, using (4.9) (on $\beta$ ), and finally integrating over $a$.

Proof of Theorem 4.1. Let $\rho_{-\infty}^{0, \theta}$ be the Weyl-Titchmarsh spectral measure for the selfadjoint realization of $-d^{2} / d x^{2}+V$ on $(-\infty, 0)$ with boundary condition

$$
u(0) \cos \theta-u^{\prime}(0) \sin \theta=0 .
$$

By a formula going back to work of Carmona (compare [5. Theorem III.3.6]; for a proof under the assumptions used here see [31, Appendix B]) we have for every $a$ and $b$ that

$$
d \rho_{a, b}(E)=\mathrm{w}-\lim _{N \rightarrow \infty} \frac{1}{\pi} \int_{0}^{\pi} r_{0}\left(a+D+b+N, \theta, E, V_{a, b}\right)^{-2} d \rho_{-\infty}^{0, \theta}(E) d \theta .
$$

Thus for continuous, non-negative $f$ with support in $\left(E_{0}-\varepsilon, E_{0}+\varepsilon\right)$ we have

$$
\begin{aligned}
& \int f(E) d \rho(E) \\
& =\int_{b_{1}}^{b_{2}} \int_{a_{1}}^{a_{2}} \int f(E) d \rho_{a, b}(E) d a d b \\
& =\int_{b_{1}}^{b_{2}} \int_{a_{1}}^{a_{2}} \lim _{N \rightarrow \infty} \frac{1}{\pi} \int_{0}^{\pi} \int f(E) r_{0}\left(a+D+b+N, \theta, E, V_{a, b}\right)^{-2} d \rho_{-\infty}^{0, \theta}(E) d \theta d a d b .
\end{aligned}
$$

The estimate (4.3) in Lemma 4.2, in particular its uniformity in $\theta, N$ and $E$, allows us to exchange the $(a, b)$-integration and the $N$-limit in (4.14) by Fatou's lemma and to conclude that

$$
\begin{aligned}
\int f(E) d \rho(E) & \leq \frac{C}{\pi} \int_{0}^{\pi} \int f(E) d \rho_{-\infty}^{0, \theta}(E) d \theta \\
& =C \int f(E) d E
\end{aligned}
$$

where finally spectral averaging over $\theta$ was used, see [19].

It remains to comment on the changes, in fact simplifications, in the above proof which show that $\rho$ is absolutely continuous on $(0, \infty)$. One has $\frac{d}{d a} \phi_{0}(a, \theta, E, 0)=$ $\cos ^{2} \phi_{0}+E \sin ^{2} \phi_{0}>0$ for all $E>0, \theta$ and $a$. (No singular $\theta$-values!) Thus, if $0<E_{1}<E_{2}<\infty$, then by continuity $\frac{d}{d a} \phi_{0}(a, \theta, E, 0) \geq C>0$ uniformly in $E \in\left[E_{1}, E_{2}\right], \theta \in[0, \pi)$, and $a \in\left[a_{1}, a_{2}\right]$. Proceeding as in the proof of Lemma 4.2 integrating with respect to first $a$ and then $b$, yields (4.3) uniformly in $N>0$, $\theta \in[0, \pi)$, and $E \in\left[E_{1}, E_{2}\right]$. This implies local Lipshitz continuity of $\rho$ in $(0, \infty)$ as before. 
Proof of Theorem 2.2 in the case $X=P$. Let $\left(\xi_{n}\right)_{n \in \mathbb{Z} \backslash\{0,1\}}=\left(p_{n}\right)_{n \in \mathbb{Z} \backslash\{0,1\}}$ be fixed and such that $\left|M_{s c}\right|=0$, and let $p_{0} \geq s$ and $p_{1} \geq 2 s$. Then $H_{\xi}^{P}=H_{a, b}$, where the potential $V$ in (3.1) is determined by $\left(p_{n}\right)_{n \in \mathbb{Z} \backslash\{0,1\}}$ and $a=p_{0}-s$, $b=p_{1}-2 s, D=2 s, q(x)=f(x-s)$.

If $M_{0}$ is the set from Theorem 4.1 then $M:=M_{0} \cup\{0\}$ is discrete. Pick arbitrary $a_{2}>a_{1}>0$ and $b_{2}>b_{1}>0$. Then by Theorem 4.1

$$
\int_{b_{1}}^{b_{2}} \int_{a_{1}}^{a_{2}} \rho_{a, b}\left(M_{s c} \cap(\mathbb{R} \backslash M)\right) d a d b=\rho\left(M_{s c} \cap(\mathbb{R} \backslash M)\right)=0 .
$$

Thus $\rho_{a, b}\left(M_{s c} \cap(\mathbb{R} \backslash M)\right)=0$ for (Lebesgue) almost every $(a, b) \in\left[a_{1}, a_{2}\right] \times\left[b_{1}, b_{2}\right]$. We infer from (2.5) that $\rho_{a, b}^{s c}(\mathbb{R} \backslash M)=0$, and thus $\sigma_{s c}\left(H_{a, b}\right) \cap(\mathbb{R} \backslash M)=\emptyset$ for almost every $(a, b) \in\left[a_{1}, a_{2}\right] \times\left[b_{1}, b_{2}\right]$. Since $p_{0}$ and $p_{1}$ have absolutely continuous distribution with density $\alpha e^{-\alpha t}$, it follows that

$$
\mathbb{P}^{0,1}\left\{\sigma_{s c}\left(H_{\xi}^{P}\right) \cap(\mathbb{R} \backslash M)=\emptyset\right\} \geq \int_{a_{1}+s}^{a_{2}+s} \alpha e^{-\alpha t} d t \cdot \int_{b_{1}+2 s}^{b_{2}+2 s} \alpha e^{-\alpha t} d t>0 .
$$

This implies (2.6) since $\mathbb{R} \backslash M$ is open.

\section{Spectral Averaging for the Displacement Model}

To prove Theorem 2.2 in the case $X=D$ we aim at Theorem 5.4 below, which is an analog to Theorem 4.1. However, the statements and proofs are somewhat more involved. Again, we exclude a set $M$ of "singular" energies, see (5.1) below. For $E_{0} \in \mathbb{R} \backslash M$ we will establish spectral averaging in a neighborhood of $E_{0}$ for the family $-d^{2} / d x^{2}+\tilde{V}_{a, b}$ defined by (5.7) below. The averaging will be done over $(a, b) \in\left[a_{0}-\delta, a_{0}+\delta\right] \times\left[b_{0}-\delta, b_{0}+\delta\right]$ with $a_{0}, b_{0} \in(0,1-D)$. By picking $\delta>0$ sufficiently small we can guarantee that for every initial angle $\theta$ either the Prüfer angle $\phi_{0}\left(1, \theta, \tilde{V}_{a, b}\right)$ is non-stationary in $a$ or $\phi_{0}\left(2, \theta, \tilde{V}_{a, b}\right)$ is non-stationary in $b$ (for fixed $a$ ). In fact, this will require that we exclude a finite set (depending on $E_{0}$ and $a_{0}$ ) of possible choices for $b_{0}$, see Lemma 5.2 below. After these preparations, Lemma 5.3 below will be proven in the same way as the corresponding Lemma 4.2 in the Poisson case.

Let $q \in L^{1}(0, D)$ be real valued and non-zero, and $u_{0}(\cdot, \theta, E, q)$ the solution defined in Corollary [3.2. The set

$$
\begin{aligned}
M:= & \left\{E>0: u_{0}^{\prime}(D, \theta, E, q)^{2}+E u_{0}(D, \theta, E, q)^{2}\right. \\
= & \left.\cos ^{2} \theta+E \sin ^{2} \theta \text { for all } \theta \in \mathbb{R}\right\} \cup\{0\} \\
\cup\{ & E<0: \text { There is a solution } u \text { of }-u^{\prime \prime}+q u=E u \text { such that } \\
& \left.\frac{u^{\prime}(0)}{u(0)}, \frac{u^{\prime}(D)}{u(D)} \in\{-\sqrt{|E|}, \sqrt{|E|}\}\right\}
\end{aligned}
$$

is discrete in $\mathbb{R}$ because $M \cap(-\infty, 0]$ is discrete in $(-\infty, 0]$ by Theorem 3.1 and $M \cap[0, \infty)$ is discrete by Corollary [3.2. Note that $M_{1} \subset M$, where $M_{1}$ is the set from Corollary 3.2 ,

Let the Prüfer amplitude $r_{c}(x, \theta, E, Q)$ and the Prüfer angle $\phi_{c}(x, \theta, E, Q)$ be defined as in Section 4. We will often drop the parameter $E$. For $0<D<1$, 
$q \in L^{1}(0, D)$ real-valued and non-zero, and $a \in(0,1-D)$ let

$$
\tilde{V}_{a}(x):= \begin{cases}q(x-a), & a \leq x \leq a+D \\ 0, & \text { elsewhere in }[0,1] .\end{cases}
$$

\section{Lemma 5.1.}

$$
\begin{aligned}
\frac{d}{d a} \phi_{0}\left(1, \theta, \tilde{V}_{a}\right)= & -\cos ^{2} \phi_{0}\left(1, \theta, \tilde{V}_{a}\right)-E \sin ^{2} \phi_{0}\left(1, \theta, \tilde{V}_{a}\right) \\
& +\frac{\cos ^{2} \phi_{0}(a, \theta, 0)+E \sin ^{2} \phi_{0}(a, \theta, 0)}{r_{a}^{2}\left(1, \phi_{0}(a, \theta, 0), \tilde{V}_{a}\right)} .
\end{aligned}
$$

Proof. We have

$$
\begin{aligned}
\phi_{0}\left(1, \theta, \tilde{V}_{a}\right) & =\phi_{a+D}\left(1, \phi_{0}\left(D, \phi_{0}(a, \theta, 0), q\right), 0\right) \\
& =\phi_{\alpha(a)}(1, \beta(a), 0),
\end{aligned}
$$

where $\alpha(a)=a+D$ and $\beta(a)=\phi_{0}\left(D, \phi_{0}(a, \theta, 0), q\right)$. Setting also $f(\alpha, \beta)=$ $\phi_{\alpha}(1, \beta, 0)$, we get

$$
\frac{d}{d a} \phi_{0}\left(1, \theta, \tilde{V}_{a}\right)=\frac{d}{d a} f(\alpha(a), \beta(a))=\frac{\partial f}{\partial \alpha}+\beta^{\prime} \frac{\partial f}{\partial \beta} .
$$

By a well known formula (e.g. [32, Proposition 11])

$$
\frac{\partial f}{\partial \beta}=\frac{1}{r_{\alpha}^{2}(1, \beta, 0)} \text {. }
$$

We have $\frac{d}{d a} \phi_{0}(a, \theta, 0)=\cos ^{2} \phi_{0}(a, \theta, 0)+E \sin ^{2} \phi_{0}(a, \theta, 0)$. This and the argument used in (5.4) yields

$$
\beta^{\prime}(a)=\frac{\cos ^{2} \phi_{0}(a, \theta, 0)+E \sin ^{2} \phi_{0}(a, \theta, 0)}{r_{0}^{2}\left(D, \phi_{0}(a, \theta, 0), q\right)} .
$$

Finally, to calculate $\partial f / \partial \alpha$, we use the equality

$$
\phi_{\alpha}(1, \beta, 0)=-\phi_{1}(\alpha, \pi-\beta, 0) \quad(\bmod \pi) .
$$

Thus,

$$
\begin{aligned}
\frac{\partial f}{\partial \alpha} & =-\cos ^{2} \phi_{1}(\alpha, \pi-\beta, 0)-E \sin ^{2} \phi_{1}(\alpha, \pi-\beta, 0) \\
& =-\cos ^{2} \phi_{\alpha}(1, \beta, 0)-E \sin ^{2} \phi_{\alpha}(1, \beta, 0) .
\end{aligned}
$$

Inserting (5.4), (5.5) and (5.6) in (5.3) concludes the proof.

Let $0<D<1, q \in L^{1}(0, D)$ real valued and non-zero, and $a, b \in(0,1-D)$. Let

$$
\tilde{V}_{a, b}(x)= \begin{cases}q(x-a) & \text { for } a \leq x \leq a+D, \\ q(x-1-b) & \text { for } 1+b \leq x \leq 1+b+D, \\ 0 & \text { elsewhere in }[0,2] .\end{cases}
$$

Fix an $E_{0} \in \mathbb{R} \backslash M$. Also fix $a \in(0,1-D)$.

Lemma 5.2. There exists a finite subset $M\left(a, E_{0}\right) \subset(0,1-D)$ with the following property:

If $b \in(0,1-D) \backslash M\left(a, E_{0}\right)$, then there exist closed sets $T_{1} \subset \mathbb{R}$ and $T_{2} \subset \mathbb{R}$ such that

(i) $T_{1} \cup T_{2}=\mathbb{R}$

(ii) $\frac{d}{d a} \phi_{0}\left(1, \theta, \tilde{V}_{a}, E_{0}\right) \neq 0$ for all $\theta \in T_{1}$,

(iii) $\frac{d}{d b} \phi_{0}\left(2, \theta, \tilde{V}_{a, b}, E_{0}\right) \neq 0$ for all $\theta \in T_{2}$. 
Proof. Lemma 5.1 and $r_{0}\left(1, \theta, \tilde{V}_{a}\right)=r_{0}(a, \theta, 0) r_{a}\left(1, \phi_{0}(a, \theta, 0), \tilde{V}_{a}\right)$ imply that

$$
\begin{gathered}
\frac{d}{d a} \phi_{0}\left(1, \theta, \tilde{V}_{a}\right)=0 \\
\Longleftrightarrow \quad u_{0}^{\prime}\left(D, \phi_{0}(a, \theta, 0), q\right)^{2}+E_{0} u_{0}\left(D, \phi_{0}(a, \theta, 0), q\right)^{2} \\
=\cos ^{2} \phi_{0}(a, \theta, 0)+E_{0} \sin ^{2} \phi_{0}(a, \theta, 0),
\end{gathered}
$$

where we also used the fact that $u_{0}^{\prime}\left(\cdot, \theta, \tilde{V}_{a}\right)^{2}+E_{0} u_{0}\left(\cdot, \theta, \tilde{V}_{a}\right)^{2}$ is constant in $[a+D, 1]$.

Since $E_{0} \in \mathbb{R} \backslash M$ and therefore $E_{0} \in \mathbb{R} \backslash M_{1}$, it follows from analyticity in $\alpha$ that

$$
N\left(E_{0}\right):=\left\{\alpha \in \mathbb{R}: \quad u_{0}^{\prime}(D, \alpha, q)^{2}+E_{0} u_{0}(D, \alpha, q)^{2}=\cos ^{2} \alpha+E_{0} \sin ^{2} \alpha\right\}
$$

is a discrete and, obviously, $\pi$-periodic subset of $\mathbb{R}$. The function $\alpha(\theta)=\phi_{0}(a, \theta, 0)$ is strictly increasing in $\theta$ and such that $\alpha(\theta+\pi)=\alpha(\theta)+\pi$ for all $\theta$. This implies that

$$
N\left(a, E_{0}\right):=\left\{\theta \in \mathbb{R}: \phi_{0}(a, \theta, 0) \in N\left(E_{0}\right)\right\}
$$

is discrete and $\pi$-periodic. By (5.8) we have

$$
\frac{d}{d a} \phi_{0}\left(1, \theta, \tilde{V}_{a}\right) \neq 0 \quad \text { for } \theta \in \mathbb{R} \backslash N\left(a, E_{0}\right) .
$$

Let

$$
M\left(a, E_{0}\right):=\left\{b \in(0,1-D): \frac{d}{d b} \phi_{0}\left(2, \theta, \tilde{V}_{a, b}\right)=0 \text { for some } \theta \in N\left(a, E_{0}\right)\right\} .
$$

As in Lemma 5.1, one gets

$$
\begin{aligned}
\frac{d}{d b} \phi_{0}\left(2, \theta, \tilde{V}_{a, b}\right)= & -\cos ^{2} \phi_{0}\left(2, \theta, \tilde{V}_{a, b}\right)-E_{0} \sin ^{2} \phi_{0}\left(2, \theta, \tilde{V}_{a, b}\right) \\
& +\frac{u_{0}^{\prime}\left(1+b, \theta, \tilde{V}_{a}\right)^{2}+E_{0} u_{0}\left(1+b, \theta, \tilde{V}_{a}\right)^{2}}{r_{0}^{2}\left(2, \theta, \tilde{V}_{a, b}\right)},
\end{aligned}
$$

and as in (5.8) this implies

$$
\begin{gathered}
\frac{d}{d b} \phi_{0}\left(2, \theta, \tilde{V}_{a, b}\right)=0 \\
\Longleftrightarrow \quad u_{0}^{\prime}\left(D, \phi_{0}(b, \eta, 0), q\right)^{2}+E_{0} u_{0}\left(D, \phi_{0}(b, \eta, 0), q\right)^{2} \\
=\cos ^{2} \phi_{0}(b, \eta, 0)+E_{0} \sin ^{2} \phi_{0}(b, \eta, 0),
\end{gathered}
$$

where $\eta=\phi_{0}\left(1, \theta, \tilde{V}_{a}\right)$. This holds if and only if $\phi_{0}(b, \eta, 0) \in N\left(E_{0}\right)$. For fixed $\theta$ and thus fixed $\eta$ the set

$$
\left\{b \in \mathbb{R}: \phi_{0}(b, \eta, 0) \in N\left(E_{0}\right)\right\}
$$

is discrete. (This is clear for $E_{0}>0$. If $E_{0}<0$ then either $\phi_{0}(b, \eta, 0)$ is strictly monotonic in $b$ or $\phi_{0}(b, \eta, 0)=\eta$ constantly in $b$. The latter would require $\cot \eta \in$ $\pm \sqrt{\left|E_{0}\right|}$ and cannot happen in combination with $\phi_{0}(b, \eta, 0) \in N\left(E_{0}\right)$, since (5.11) would yield a contradiction to $E_{0} \notin M$.) From this and $\pi$-periodicity one gets that

$$
\tilde{M}\left(a, E_{0}\right):=\left\{b \in \mathbb{R}: \phi_{0}\left(b, \phi_{0}\left(1, \theta, V_{a}\right), 0\right) \in N\left(E_{0}\right) \quad \text { for some } \theta \in N\left(a, E_{0}\right)\right\}
$$

is discrete. Thus $M\left(a, E_{0}\right)=\tilde{M}\left(a, E_{0}\right) \cap(0,1-D)$ is finite.

For $b \in(0,1-D) \backslash M\left(a, E_{0}\right)$ one has $\frac{d}{d b} \phi_{0}\left(2, \theta, \tilde{V}_{a, b}\right) \neq 0$ for all $\theta \in N\left(a, E_{0}\right)$. Continuity and periodicity of $\frac{d}{d b} \phi_{0}\left(2, \theta, \tilde{V}_{a, b}\right)$ in $\theta$ (compare (5.10) $)$ imply the existence of $\varepsilon>0$ such that $\frac{d}{d b} \phi_{0}\left(2, \theta, \tilde{V}_{a, b}\right) \neq 0$ for all $\theta \in T_{2}:=\left\{\theta: \operatorname{dist}\left(\theta, N\left(a, E_{0}\right)\right) \leq\right.$ 
$\varepsilon\}$. By (5.9) one has $\frac{d}{d a} \phi_{0}\left(1, \theta, \tilde{V}_{a}\right) \neq 0$ for $\theta \in T_{1}:=\left\{\theta: \operatorname{dist}\left(\theta, N\left(a, E_{0}\right)\right) \geq \varepsilon\right\}$. This completes the proof, since $T_{1}$ and $T_{2}$ are closed with $T_{1} \cup T_{2}=\mathbb{R}$.

Let $V \in L_{l o c}^{1}(\mathbb{R})$ be real-valued and such that $-\frac{d^{2}}{d x^{2}}+V$ is limit point at $+\infty$ and $-\infty$. Extend the definition of $\tilde{V}_{a, b}$ in (5.7) to the whole line by setting $\tilde{V}_{a, b}(x)=$ $V(x)$ for $x<0$ and for $x>2$.

Lemma 5.3. Fix $E_{0} \in \mathbb{R} \backslash M$. Let $a_{0} \in(0,1-D)$ and $b_{0} \in(0,1-D)$ be given such that there exist closed sets $T_{1} \subset \mathbb{R}$ and $T_{2} \subset \mathbb{R}$ which satisfy (i), (ii) and (iii) of Lemma 5.2. Then there exist $\delta>0, \varepsilon>0$ and $C>0$ such that

$$
\int_{a_{0}-\delta}^{a_{0}+\delta} \int_{b_{0}-\delta}^{b_{0}+\delta} r_{0}\left(N, \theta, E, \tilde{V}_{a, b}\right)^{-2} d b d a \leq C
$$

uniformly in $N>0, \theta \in[0, \pi)$ and $E \in\left[E_{0}-\varepsilon, E_{0}+\varepsilon\right]$.

Proof. It is sufficient to assume $N>2$. We will treat the two cases $\theta \in T_{1}$ and $\theta \in T_{2}$ separately. First, assume $\theta \in T_{1}$.

Similarly to (4.4), we have

$$
\begin{aligned}
r_{0}\left(N, \theta, \tilde{V}_{a, b}\right)^{-2} & =r_{0}\left(1, \theta, \tilde{V}_{a}\right)^{-2} r_{1}\left(N, \phi_{0}\left(1, \theta, \tilde{V}_{a}\right), \tilde{V}_{a, b}\right)^{-2} \\
& \leq C_{1} r_{0}\left(N-1, \phi_{0}\left(1, \theta, \tilde{V}_{a}\right), \tilde{V}_{b}\right)^{-2}
\end{aligned}
$$

locally uniformly in $(a, \theta, E)$. Let $\alpha:=\phi_{0}\left(1, \theta, \tilde{V}_{a}\right)$. Then $\frac{d \alpha}{d a}=\frac{d}{d a} \phi_{0}\left(1, \theta, \tilde{V}_{a}\right) \neq 0$ at $\left(a_{0}, E_{0}\right)$. Continuity in $(a, \theta, E)$ implies that there exist $\varepsilon>0$ and $\delta>0$ such that

$$
\left|\frac{d \alpha}{d a}\right| \geq C_{2}>0
$$

uniformly in $\theta \in T_{1}, E \in\left[E_{0}-\varepsilon, E_{0}+\varepsilon\right]$ and $a \in\left[a_{0}-\delta, a_{0}+\delta\right]$. Substituting $\alpha$ for $a$ and using the argument from the proof of (4.8) and 4.10), we conclude that

$$
\int_{b_{0}-\delta}^{b_{0}+\delta} \int_{a_{0}-\delta}^{a_{0}+\delta} r_{0}\left(N, \theta, \tilde{V}_{a, b}\right)^{-2} d a d b \leq C
$$

uniformly in $N>2, \theta \in T_{1}$ and $E \in\left[E_{0}-\varepsilon, E_{0}+\varepsilon\right]$.

Next, consider the case $\theta \in T_{2}$, in which we will do the $b$-integration in (5.12) first. We have

$$
\begin{aligned}
r_{0}\left(N, \theta, \tilde{V}_{a, b}\right)^{-2} & =r_{0}\left(2, \theta, \tilde{V}_{a, b}\right)^{-2} r_{2}\left(N, \phi_{0}\left(2, \theta, \tilde{V}_{a, b}\right), \tilde{V}_{a, b}\right)^{-2} \\
& \leq C_{3} r_{0}\left(N-2, \phi_{0}\left(2, \theta, \tilde{V}_{a, b}\right), V(\cdot+2)\right)^{-2}
\end{aligned}
$$

locally uniformly in $(a, b, \theta, E)$. Setting $\beta(b):=\phi_{0}\left(2, \theta, \tilde{V}_{a, b}\right)$, we see that $\frac{d \beta}{d b} \neq 0$ at $\left(a_{0}, b_{0}, E_{0}\right)$. We now use continuity of $\frac{d}{d b} \phi_{0}\left(2, \theta, \tilde{V}_{a, b}\right)$ in $(a, b, \theta, E)$ and continue to argue very similarly to the case $\theta \in T_{1}$, doing the $b$-integration first. We conclude the existence of $\varepsilon>0$ and $\delta>0$ such that

$$
\int_{a_{0}-\delta}^{a_{0}+\delta} \int_{b_{0}-\delta}^{b_{0}+\delta} r_{0}\left(N, \theta, \tilde{V}_{a, b}\right)^{-2} d b d a \leq C^{\prime}<\infty
$$

uniformly in $N>2, \theta \in T_{2}$ and $E \in\left[E_{0}-\varepsilon, E_{0}+\varepsilon\right]$.

This implies (5.12) with the smaller of the two values found for $\varepsilon$ respectively $\delta$ and the larger of the constants $C$ and $C^{\prime}$.

Finally, denoting by $\tilde{\rho}_{a, b}$ the spectral measure of $-d^{2} / d x^{2}+\tilde{V}_{a, b}$, the next result follows from Lemma 5.3 in the same way as Theorem 4.1 followed from Lemma 4.2 
Theorem 5.4. For $E_{0} \in \mathbb{R} \backslash M, a_{0}, b_{0}, \delta$ and $\varepsilon$ as in Lemma[5.3, and Borel sets $B$, define

$$
\tilde{\rho}(B)=\int_{a_{0}-\delta}^{a_{0}+\delta} \int_{b_{0}-\delta}^{b_{0}+\delta} \tilde{\rho}_{a, b}(B) d b d a .
$$

Then the Borel measure $\tilde{\rho}$ is absolutely continuous in $\left(E_{0}-\varepsilon, E_{0}+\varepsilon\right)$.

Proof of Theorem 2.2 for $X=D$. Let $\left(\xi_{n}\right)_{n \in \mathbb{Z} \backslash\{0,1\}}=\left(d_{n}\right)_{n \in \mathbb{Z} \backslash\{0,1\}}$ be fixed so that $\left|M_{s c}\right|=0$. Then for arbitrary $d_{0}, d_{1} \in\left[-d_{\max }, d_{\max }\right]$ the operator $H_{\xi}^{D}$ is, up to translation by $1 / 2$, given by $-d^{2} / d x^{2}+\tilde{V}_{a, b}$, where $\tilde{V}_{a, b}(x)=\sum_{n} f\left(x-\frac{1}{2}-n-d_{n}\right)$ for $x \in \mathbb{R} \backslash[0,2]$ and $\tilde{V}_{a, b}(x)$ is defined by (5.7) with $q(x):=f\left(x-\left(\frac{1}{2}-d_{\text {max }}\right)\right)$, $a:=d_{\max }+d_{0}, b:=d_{\max }+d_{1}$ if $x \in[0,2]$.

Let $M$ be the discrete set defined in (5.1), and let $E_{0} \in \mathbb{R} \backslash M$. Let $\mu$ be the distribution of the random variable $d_{0}$ or $d_{1}$, and $\mu_{a c}$ its absolutely continuous part. Pick $a_{0}$ such that $a_{0}-d_{\max } \in \operatorname{supp} \mu_{a c}$ and $b_{0}$ such that $b_{0} \notin M\left(a_{0}, E_{0}\right)$, the finite set from Lemma 5.2 and $b_{0}-d_{\max } \in \operatorname{supp} \mu_{a c}$. This is possible since $\operatorname{supp} \mu_{a c}$ is infinite.

Lemma 5.2 shows that Lemma 5.3 and thus Theorem 5.4 apply with this choice of $E_{0}, a_{0}$ and $b_{0}$. Thus the measure $\tilde{\rho}$ defined by (5.14) is absolutely continuous in $\left(E_{0}-\varepsilon, E_{0}+\varepsilon\right)$ for suitable $\varepsilon>0$ and $\delta>0$. Thus $\tilde{\rho}_{a, b}\left(M_{s c} \cap\left(E_{0}-\varepsilon, E_{0}+\varepsilon\right)\right)=0$ and therefore $\sigma_{s c}\left(-d^{2} / d x^{2}+\tilde{V}_{a, b}\right) \cap\left(E_{0}-\varepsilon, E_{0}+\varepsilon\right)=\emptyset$ for almost every $(a, b) \in$ $\left[a_{0}-\delta, a_{0}+\delta\right] \times\left[b_{0}-\delta, b_{0}+\delta\right]$. Since $a_{0}-d_{\max }, b_{0}-d_{\max } \in \operatorname{supp} \mu_{a c}$, this implies that $\mathbb{P}^{0,1}\left\{\sigma_{s c}\left(H_{\xi}^{D}\right) \cap\left(E_{0}-\varepsilon, E_{0}+\varepsilon\right)\right\}>0$.

\section{A SeCond proof of localization for the Poisson MOdel}

Consider a Poisson process on $\mathbb{R}$ with density $\alpha>0$ and let $I \subset \mathbb{R}$ be a nontrivial finite interval. The event that $I$ contains exactly one Poisson point $X$ has positive probability $\alpha|I| e^{-\alpha|I|}$. Conditioned on this event, the point $X$ has uniform distribution on $I$; see e.g. [13, p.352f]. Thus we have some 'displacement-type randomness' in the Poisson potential $V_{\omega}^{P}$. Using also the independence properties of the Poisson process, this allows us to apply the methods developed in the context of the displacement model and thereby to provide an alternate proof of localization for the Poisson model. We briefly outline this proof below, mainly for the reason that uniform conditional distribution of Poisson points generalizes to dimension $d>1$, while the approach of using the distances $p_{n}$ from (1.4) does not. This may provide a strategy for proving localization in the multidimensional Poisson model.

For the Poisson potential $V_{\omega}^{P}(x)=\sum_{i} f\left(x-x_{i}(\omega)\right)$ with supp $f \subset[-s, s]$ as defined in Section 1, choose $L>2 s$ and $d \in\left(0, \frac{L}{2}-s\right)$. Let $\Omega_{0} \subset \Omega$ be the event that none of the intervals $\left[-s, \frac{L}{2}-d\right],\left[\frac{L}{2}+d, \frac{3 L}{2}-d\right]$ and $\left[\frac{3 L}{2}+d, 2 L+s\right]$ contain any Poisson points, while each of the intervals $\left[\frac{L}{2}-d, \frac{L}{2}+d\right]$ and $\left[\frac{3 L}{2}-d, \frac{3 L}{2}+d\right]$ contains exactly one Poisson point. By (1.3) and independence of the number of points in disjoint intervals we have $\mathbb{P}\left(\Omega_{0}\right)>0$.

For $\omega \in \Omega_{0}$, let $X_{1}(\omega)$ and $X_{2}(\omega)$ be the unique Poisson points in $\left[\frac{L}{2}-d, \frac{L}{2}+d\right]$ and $\left[\frac{3 L}{2}-d, \frac{3 L}{2}+d\right]$, respectively.

On $\Omega_{0}$, the potentials $\left.V^{P}\right|_{\mathbb{R} \backslash[0,2 L]}$ and $\left.V^{P}\right|_{[0,2 L]}=f\left(\cdot-X_{1}\right)+f\left(\cdot-X_{2}\right)$ are independent with respect to the conditional probability $\mathbb{P}_{\Omega_{0}}$, since $f\left(\cdot-X_{1}\right)+$ $f\left(\cdot-X_{2}\right)$ does not overlap with $f\left(\cdot-x_{i}\right)$ for any of the remaining points $x_{i} \in$ $\mathbb{R} \backslash[-s, 2 L+s]$. 
Also, $X_{1}$ and $X_{2}$ are independent and uniformly distributed with respect to $\mathbb{P}_{\Omega_{0}}$, i.e. for $I_{1} \subset\left[\frac{L}{2}-d, \frac{L}{2}+d\right]$ and $I_{2} \subset\left[\frac{3 L}{2}-d, \frac{3 L}{2}+d\right]$ we have

$$
\mathbb{P}_{\Omega_{0}}\left(X_{1} \in I_{1}, X_{2} \in I_{2}\right)=\frac{\left|I_{1}\right|}{2 d} \cdot \frac{\left|I_{2}\right|}{2 d} .
$$

This allows us to apply the results of Section 5 (replacing the unit intervals $[0,1]$ and $[1,2]$ by $[0, L]$ and $[L, 2 L])$ and prove an analog of Theorem 2.2, For fixed $\left.V^{P}\right|_{\mathbb{R} \backslash[0,2 L]}$ with $\left|M_{s c}\right|=0\left(M_{s c}\right.$ the support of $\sigma_{s c}\left(H_{\omega}^{P}\right)$ for arbitrary $\left.X_{1}, X_{2}\right)$, we have

$$
\mathbb{P}^{X_{1}, X_{2}}\left\{\sigma_{s c}\left(H_{\omega}^{P}\right) \cap\left(E_{0}-\varepsilon, E_{0}+\varepsilon\right)=\emptyset\right\}>0
$$

for $E_{0} \in \mathbb{R} \backslash M$ and suitable $\varepsilon>0$. Here $\mathbb{P}^{X_{1}, X_{2}}$ is the distribution of $\left(X_{1}, X_{2}\right)$ on $\Omega_{0}$, and $M$ is the same discrete set as used in the proof of Theorem 2.2 for $X=D$ in Section 5 .

As in Section 2 it follows from positivity of the Lyapunov exponent that $\left|M_{s c}\right|=0$ for $\mathbb{P}_{\Omega_{0}}$-a.e. $\left.V^{P}\right|_{\mathbb{R} \backslash[0,2 L]}$. Thus, (6.1) and independence of $\left.V^{P}\right|_{\mathbb{R} \backslash[0,2 L]}$ and $\left.V^{P}\right|_{[0,2 L]}$ yield

$$
\mathbb{P}_{\Omega_{0}}\left\{\sigma_{s c}\left(H_{\omega}^{P}\right) \cap\left(E_{0}-\varepsilon, E_{0}+\varepsilon\right)=\emptyset\right\}>0 .
$$

We conclude that $\mathbb{P}\left\{\sigma_{s c}\left(H_{\omega}^{P}\right) \cap\left(E_{0}-\varepsilon, E_{0}+\varepsilon\right)=\emptyset\right\}>0$ since $\mathbb{P}\left(\Omega_{0}\right)>0$, implying $\Sigma_{s c}^{P}=\emptyset$ as at the end of Section 2. This completes the proof.

\section{REFERENCES}

[1] G. Borg: Eine Umkehrung der Sturm-Liouvilleschen Eigenwertaufgabe. Acta Math. 78, 1 96 (1946) MR 7:382d

[2] D. Buschmann: A proof of the Ishii-Pastur theorem by the method of subordinacy. Univ. Iagel. Acta Math. 34, 29 - 34 (1997) MR 98i:82031

[3] R. Carmona: Exponential localization in one-dimensional disordered systems. Duke Math. J. 49, 191 - 213 (1982) MR 84j:82082

[4] R. Carmona, A. Klein and F. Martinelli: Anderson localization for Bernoulli and other singular potentials. Commun. Math. Phys., 108, 41 - 66 (1987) MR 88f:82087

[5] R. Carmona and J. Lacroix: Spectral theory of random Schrödinger operators. Birkhäuser, Basel-Berlin 1990 MR 94k:47143

[6] E.A. Coddington and N. Levinson: Theory of ordinary differential equations. McGraw-Hill, New York 1955 MR 16:1022b

[7] J.-M. Combes and P.D. Hislop: Localization for some continuous random Hamiltonians in d-dimensions. J. Funct. Anal. 124, 149 - 180 (1994) MR 95g:82047

[8] J.M. Combes, P.D. Hislop and E. Mourre: Spectral averaging, perturbation of singular spectrum, and localization. Trans. Amer. Math. Soc. 348, 4883 - 4894 (1996) MR 97c:35145

[9] H. von Dreifus and A. Klein: A new proof of localization in the Anderson tight binding model. Commun. Math. Phys. 124, 285 - 299 (1989) MR 90k:82056

[10] D.J. Gilbert: On subordinacy and analysis of Schrödinger operators with two singular endpoints. Proc. Royal Soc. Edinburgh 112A, 213 - 229 (1989) MR 90j:47061

[11] I.Ya. Goldsheidt, S. Molchanov and L. Pastur: A pure-point spectrum of the stochastic onedimensional Schrödinger equation. Funct. Anal. Appl. 11, 1 - 8 (1977) [MR 57:10266

[12] A. Figotin and A. Klein: Localization of classical waves I: Acoustic waves. Commun. Math. Phys. 180, 439 - 482 (1996) MR 2000d:35240a

[13] B.V. Gnedenko: The theory of probability, 4th Edition, Chelsea Publishing Company, New York, 1967 MR 36:913

[14] W. Kirsch, S. Kotani and B. Simon: Absence of absolutely continuous spectrum for some one dimensional random but deterministic potentials. Ann. Inst. Henri Poincaré Phys. Théor. 42, 383 - 406 (1985) MR 87h:60115

[15] W. Kirsch, P. Stollmann and G. Stolz: Anderson localization for random Schrödinger operators with long range interactions. Commun. Math. Phys. 195, 495-507 (1998) MR 99e:82051 
[16] F. Klopp: Localization for semiclassical continuous random Schrödinger Operators II: The random displacement model. Helv. Phys. Acta 66, 810 - 841 (1993) MR 96c:35204

[17] F. Klopp: Localization for some continuous random Schrödinger operators. Commun. Math. Phys. 167, 553 - 569 (1995) MR 95m:82080

[18] S. Kotani: Lyapunov indices determine absolutely continuous spectra of stationary one dimensional Schrödinger operators. In: Stochastic Analysis, ed. K. Ito, 225 - 247, North Holland, Amsterdam 1984 MR 86h:60117

[19] S. Kotani: Lyapunov exponents and spectra for one-dimensional random Schrödinger operators. In: Random Matrices and their Applications, eds. J.E. Cohen, H. Kesten and C.M. Newman, American Mathematical Society, Contemporary Math. 50, 277 - 286, Providence 1986 MR 88a:60116

[20] S. Kotani: One-dimensional random Schrödinger operators and Herglotz functions. In: Probabilistic Methods in Mathematical Physics, eds. K. Ito and N. Ikeda, 219 - 250, Academic Press, Boston 1987 MR 89h:60100

[21] S. Kotani and B. Simon: Localization in general one-dimensional random systems. Commun. Math. Phys. 112, 103 - 119 (1987) MR 89d:81034

[22] H. Kunz and B. Souillard: Sur le spectre des opérateurs aux différences finies aléatoires. Commun. Math. Phys. 79, 201 - 249 (1980) MR 83f:39003

[23] N. Minami: An extension of Kotani's theorem to random generalized Sturm-Liouville operators. Commun. Math. Phys. 103, 387 - 402 (1986) MR 87j:81042

[24] N. Minami: An extension of Kotani's theorem to random generalized Sturm-Liouville operators II. In: Stochastic processes in classical and quantum systems, $411-419$, Lecture Notes in Physics 262, Springer 1986 MR 88h:81028

[25] N. Minami: Random Schrödinger operators with a constant electric field. Ann. Inst. Henri Poincaré Phys. Théor. 56, 307 - 344 (1992) MR 93d:34142

[26] S. Molchanov: Lectures on random media. In: Lectures on probability theory (Saint-Flour, 1992), 242-411, Lecture Notes in Math. 1581, Springer 1994. MR 95m:60165

[27] L. Pastur and A. Figotin: Spectra of random and almost-periodic operators. Springer-Verlag, 1991 MR 94h:47068

[28] M. Reed and B. Simon: Methods of modern mathematical physics, IV. Analysis of operators, Academic Press, New York 1978 MR 58:12429c

[29] B. Simon: Localization in general one dimensional random systems, I. Jacobi matrices. Commun. Math. Phys. 102, 327 - 336 (1985) MR 87h:81039

[30] B. Simon and T. Wolff: Singular continuous spectrum under rank one perturbations and localization for random Hamiltonians. Commun. Pure Appl. Math. 39, 75 - 90 (1986) MR 87k:47032

[31] G. Stolz: Spectral theory of Schrödinger operators with potentials of infinite barriers type. Habilitationsschrift, Frankfurt 1994

[32] G. Stolz: Localization for random Schrödinger operators with Poisson potential. Ann. Inst. Henri Poincaré Phys. Théor. 63, 297 - 314 (1995) MR 96h:34174

Fachbereich Mathematik, Johann Wolfgang Goethe-Universität, D-60054 Frankfurt, GERMANY

E-mail address: buschmann@dpg.de

Department of Mathematics, University of Alabama at Birmingham, Birmingham, AlABAMA 35294-1170

E-mail address: stolz@math.uab.edu 